\title{
That Gut Feeling: The Role of Inflammatory Cytokines in Depression Among Patients with Inflammatory Bowel Disease
}

\author{
Authors: \\ Brant J. Chapman, ${ }^{1,2} *$ Graham B. Jones ${ }^{2}$ \\ 1. Bergen Community College, Paramus, New Jersey, USA \\ 2. Clinical and Translational Science Institute, Tufts University Medical Center, Boston, \\ Massachusetts, USA \\ *Correspondence to graham.jones@tufts.edu \\ Disclosure: The authors have declared no conflicts of interest. \\ Acknowledgements: The authors dedicate this review to Warren Finkelstein MD, for his decades of \\ dedication to the inflammatory bowel disease community. The authors acknowledge \\ support from the National Center for Advancing Translational Sciences [NIH UL1 \\ TROO2544]. \\ Received: \\ 07.07.20 \\ Accepted: \\ 28.09.20 \\ Keywords: \\ Crohn's disease, cytokines, depression, inflammatory bowel disease (IBD), suicide \\ risk, TNF. \\ Citation: \\ EMJ Gastroenterol. 2020;9[1]:81-90.
}

\begin{abstract}
There is mounting evidence of an associative link between inflammatory bowel disease (IBD) and clinical depression. In the first major treatise on the eponymous disease, Burrill Crohn himself noted that: "The number of cases of ileitis that have been rescued from institutions for the treatment of mental diseases emphasises not the personality but the end results of the drain of the disease upon the psychic constitution of the sufferer." In the 70 years since that prescient statement, a high incidence of neuropsychiatric symptoms (depression, anxiety, cognitive fatigue, and sleep disorders) in patients with IBD has been frequently observed. Since patients with depression have significantly increased rates of relapse, surgery, hospitalisation, and suicide, recognising and treating depression is of paramount importance. In this narrative review, the authors will trace some of the biochemical connections between intestinal inflammation and neuropsychiatric symptoms and focus on strategies to manage both. Additionally, the authors offer a cautionary reflection on the extant need for widespread screening for depression among patients with IBD.
\end{abstract}

\section{INTRODUCTION}

In the first major treatise on the eponymous disease, Burrill Crohn himself noted that: "The number of cases of ileitis that have been rescued from institutions for the treatment of mental diseases emphasises not the personality but the end results of the drain of the disease upon the

psychic constitution of the sufferer" (emphasis added). ${ }^{1}$ In the 70 years since that prescient statement, a high incidence of neuropsychiatric symptoms (depression, anxiety, cognitive fatigue, and sleep disorders) in patients with inflammatory bowel disease (IBD) has been consistently observed. ${ }^{2-4}$ As with IBD, depression lacks clear biomarkers for definitive diagnosis, 
and frequently involves complex treatment regimens. Unfortunately, there is no parallel imaging technique to colonoscopy for the brain that is comparably simple and widely available. As a result, diagnosis and treatment of depression presents unique challenges alone, and those challenges are compounded both by systemic inflammation and by various medications. Since patients with depression have significantly increased rates of relapse, surgery, hospitalisation, ${ }^{5}$ and suicide, ${ }^{6}$ recognising and treating depression is of paramount importance. In this narrative review, the authors will trace some of the biochemical connections between intestinal inflammation and neuropsychiatric symptoms and focus on strategies to manage both.

\section{COMMON THREADS: CYTOKINES}

To illuminate the connections between the immune system, peripheral inflammation, and neuropsychiatric effects, it is instructive to observe the psychological effects when systemic inflammation is induced by an immediate immune challenge. One common method for inflammation induction is injection of Salmonella abortus equi endotoxin. ${ }^{7}$ In healthy volunteers, endotoxin rapidly activates the host-defence system, inducing both TNFa and IL-6 (see below), resulting in a constellation of disrupted neurological functions referred to as 'sickness behaviour'. Among the disruptions are extraintestinal symptoms well known to legions of those with IBD: anxiety, depressed mood, fatigue, and memory impairment. In the context of treating IBD, it is important to note that both the anxiety and depressed mood normalised in parallel with decreases in the cytokine concentrations. Similar mood effects are seen with Salmonella Typhii vaccine ${ }^{8}$ and Bacillus Calmette-Guérin vaccine, ${ }^{9}$ with concomitant elevation of the same cytokines. It is also worth remembering that these were healthy volunteers, subjected to a short-duration, low-dose immune challenge in which both the cytokine levels and neuropsychiatric symptoms typically resolve in a timescale of hours. By contrast, patients with IBD have chronic immune activation, and frequently higher circulating amounts of those same cytokines, even in remission. ${ }^{10}$ Patients with IBD are, therefore, subject to prolonged alterations in brain function leading to anhedonia, anxiety, and cognitive fatigue..$^{2-4}$

Perhaps the most insightful example for this discussion is exogenous IFNa, used for both chemotherapy and hepatitis $\mathrm{C}$. There are two advantages to using IFNa therapy as a comparative study. First, given the widespread use of IFNa, there is a large dataset with robust documentation of systemic effects and mechanisms in both animals and humans. Second, owing to repeated and higher dosings over weeks and months, IFNa treatment more closely aligns with the chronic inflammatory state typically presented in IBD. As above, IFNa induces a rapid and sustained increase in other inflammatory cytokines, particularly TNFa and IL-6." Significantly, up to $50 \%$ of patients being treated with IFNa experience major depressive symptoms, with $80 \%$ reporting sickness

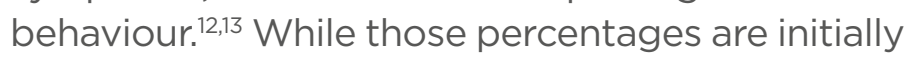
alarming, numerous large studies of patients with IBD have estimated between 20 and 45\% of patients with IBD experienced at least some depressive symptoms, ${ }^{14}$ and elevated rates of depressive disorder of $15 \%$ or higher. $^{2}$

\section{TNFa}

TNFa has been ascribed a central role in chronic inflammation not only in IBD, but also rheumatoid arthritis (RA), plaque psoriasis, psoriatic arthritis, ankylosing spondylitis, and systemic lupus erythematosus. TNFa interacts with the Type 2 receptor, leading to an inflammatory cascade via NF- $\kappa B$ activation. NF- $k B$ is a transcription factor for an enormous number of inflammatory genes, ${ }^{15,16}$ including virtually every molecule discussed in this review, including itself.

Along with, and because of, the dysregulation of so many critical factors for neurotransmission, it is little surprise that circulating TNFa is strongly associated with a variety of psychiatric effects, including depression, anxiety, cognitive fatigue, and sleep disturbances. $7,8,11,17-21$

Serum TNFa levels have been found to be significantly elevated in patients in the acute phase of major depressive disorder (MDD).17 While levels decreased during antidepressant treatment, they remained elevated in the chronic phase of MDD. Similarly, post-mortem examination of individuals with depression has found elevated levels of TNFa in a variety of 
areas of the brain. ${ }^{18}$ Perhaps unsurprisingly, both TNFa protein and mRNA were also elevated in the prefrontal cortex of teenage suicide victims, ${ }^{19}$ as well as in plasma of some adults attempting suicide. ${ }^{20}$ In clinical trials, systemic TNFa infusion was tested for proapoptotic activity with certain cancers. ${ }^{22}$ Many patients developed dose-dependent, reversible, attentional deficits, memory disorders, fatigue, confusion, and in some cases neurotoxicity.

In 1998 the U.S. Food and Drug Administration (FDA) approved infliximab for Crohn's disease, the first biologic inhibitor for TNFa, heralding a new age in the treatment of IBD. Almost overnight, anecdotal reports of rapid antidepressant effects surfaced, with later imaging studies demonstrating observable changes in brain function in 48 hours, ${ }^{21}$ and biochemical changes within 24 hours. ${ }^{23}$ As a result, in 2013, a clinical trial of infliximab for treatment-resistant depression was undertaken. ${ }^{24}$ While inhibition of TNFa was not shown to be uniformly effective, it was effective in a subgroup of patients with higher levels of C-reactive protein (CRP). This trial suggested that depression is not purely an inflammatory process, but is certainly exacerbated by existing inflammation, and treatable by suppressing inflammatory cytokines, adding to the results observed in IBD, RA, and psoriasis. ${ }^{25}$

Notably, antidepressant effects of TNF blockade are not exclusive to infliximab, nor even to antibodies. Thalidomide, which acts as a small-molecule inhibitor of TNFa, also shows antidepressant effects. ${ }^{26}$

\section{IL-6}

Along with TNFa, IL-6 is elevated in virtually all autoimmune inflammatory disorders, and has a strong association with depression. ${ }^{27}$ Among its pleiotropic roles are induction of $\mathrm{CRP}^{28}$ weakening of tight junctions at the intestinal epithelial barrier and the blood-brain barrier (BBB), ${ }^{28}$ and differentiation of $T$ helper cells into Th17 (IL-17 producing) cells. IL-6 expression

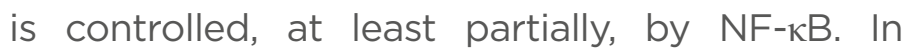
Crohn's patients, IL-6 is highly upregulated in active disease, and is strongly downregulated in infliximab responders. ${ }^{29}$ In animal studies, IL-6 has been shown to directly pass through the $\mathrm{BBB}$ and into the brain. ${ }^{30}$ As with TNFa, average IL-6 levels have been found to be elevated in the brains of successful suicides and in the cerebrospinal fluid of attempters. There is a rich literature demonstrating the strong relationship between IL-6 and dopamine in the symptoms of anhedonia, 27,28,31,32 a core symptom of MDD. Even a comparably low level of IL-6 is strongly tied to motivational effort, ${ }^{32}$ and as such is a likely contributor to the high degree of treatment nonadherence observed in IBD patients. ${ }^{33}$

Unlike TNFa, where exogenous cytokine was tested only on ill patients, the effects of IL- 6 have been directly tested by subcutaneous injection of the recombinant protein into healthy volunteers. ${ }^{34}$ Within 4 hours, self-reported measures of concentration, self-reliance, and 'high spirits' had all dropped significantly, while fatigue, sadness, and anxiety had all increased. Sleep analysis of this same cohort showed a significant decrease in time spent in the rapid eye movement phase of sleep. As emphasised above, these were healthy volunteers with a single lowdose acute stimulus over a short time period. As with TNFa, it appears that a significant portion of patients with IBD do not have sufficient biochemical adjustment to increased IL- 6 levels, and thereby experience more chronic versions of the short-term effects listed above, manifested in sleep and mood disorders.

Given early success in blocking TNFa and subsequent focus on more gut-specific inflammation (vedolizumab), IL-6 inhibitors have not yet been used extensively in IBD despite successful clinical trials. However, studies in RA have shown that blockage of the IL- 6 signalling (either antibodies to IL-6 ${ }^{35}$ or to the IL-6 receptor ${ }^{36}$ ) has positive effects on depression and anxiety symptoms, as well as improvements to sleep.

\section{IL-17A}

Of the cytokines commonly associated with both IBD and depression, IL-17A is particularly enigmatic. Th17 cells are induced either by IL- 6 or by IL-23, leading to the production of IL17A. Similar to IL-6, IL-17A is directly involved in weakening both the intestinal epithelial barrier and the BBB. In the brain, IL-17A is associated with a host of neurological diseases, including multiple sclerosis, ischaemic brain injury, and Alzheimer's disease. ${ }^{37}$ IL-17A also appears to directly induce microglia into the reactive state, leading to significant monoamine neurotransmitter issues 
(see below). ${ }^{38}$ One IL-17A inhibitor in particular, Ixekizumab, demonstrated parallel results to those mentioned above in improving depressive symptoms in patients with plaque psoriasis. ${ }^{39}$ Despite initial failure as a therapeutic target in Crohn's disease, IL-17A is still a potentially useful biomarker because IL-17A levels are associated with antidepressant effectiveness ${ }^{40}$ and even with infliximab response. ${ }^{41}$

\section{Other Targets}

While not as frequently implicated in depression, both IL-12 and IL-23 have been associated with depressive symptoms. Inhibition of both IL-12/IL23 (ustekinumab) $^{42}$ or IL-23 alone (guselkumab) ${ }^{43}$ improve depression and anxiety scores. Somewhat more surprising is the finding that vedolizumab, an a4ß7-integrin inhibitor acting virtually exclusively in the gut, also ameliorates depression and anxiety, and improves sleep quality. ${ }^{44}$ In fact, a 2016 meta-analysis showed that inhibition of virtually any single cytokine involved in inflammation improved symptoms of depression. ${ }^{25}$

\section{TRANSMISSION TO THE BRAIN}

There are three distinct pathways for transmission of inflammation from the intestines to the brain: the neural, the humoral, and the cellular. It is very likely that all three are in play with IBD.

In the neural pathway, cytokines bind directly to afferent fibres of the vagus nerve, transmitting the signal directly to the brain. Since this signalling does not require any molecular transport across the BBB, the transmission is fastest. There is a wealth of data on direct vagus stimulation (albeit not from the intestines) for treatment-resistant depression. ${ }^{45}$

In the humoral pathway, cytokines and other molecular miscreants directly cross the BBB into the brain, either passively or by active transport. This is perhaps the best studied of the three pathways, because BBB leakage is implicated in any number of neurological disorders, and it becomes more pronounced with age; 46 while active transport of cytokines and other immunomodulators is partly circadian, and is associated with sleep disruptions. ${ }^{47}$ It is worth noting that the epithelial barriers of the intestine, the retina, and the brain are similarly constituted and disrupted by many of the same factors. ${ }^{48}$ For example, Calarge et al. ${ }^{49}$ were recently able to show that increased intestinal permeability is directly associated with depressive symptoms, specifically in unmedicated adolescents. This aligns well with other data demonstrating that both IL- 6 and IL-17 alter the integrity of the BBB from the periphery, while activated microglia also act to disrupt the BBB and express cytokines and chemokines from the inside..$^{50}$

At least partially, the humoral pathway opens the door to the cellular pathway, both by loosening the tight-junction regulation and by increased expression of monocyte chemotactic protein (MCP-1/CCL2O) in brain endothelial cells, thereby permitting cell trafficking of monocytes and macrophages into the brain. ${ }^{51}$

\section{EFFECTS ON MONOAMINE NEUROTRANSMITTERS}

Mechanistically speaking, the effects of inflammatory cytokines represent a form of 'perfect storm' for effects on the brain, and specifically on the synthesis and availability of monoamine neurotransmitters. Figure 1 and 2 illustrate a number of the pathways leading to neurological effects of inflammation.

\section{The Indole-2,3-dioxygenase pathway: The Monoamine Crossroads}

One of the most well documented alterations in neurotransmitter levels is brought about by indole-2,3-dioxygenase (IDO1), a uniquely destructive nexus in the synthesis, signalling, and release of monoamines. As shown in Figure 1, this enzyme catabolises tryptophan, thereby inhibiting serotonin synthesis, increases excitotoxic glutamatergic signalling and glutamate release, and in turn hinders dopamine release. When it was discovered, IDO1 was thought to be an intestinal variation of the tryptophan-2,3-dioxygenase (expressed primarily in the liver). Later studies demonstrated that IDO1 is induced by cytokines and transcribed by $N F-\kappa B$. One proposed rationale for this particular enzyme is an evolutionary defence against pathogens by lowering the amount of available tryptophan, with depressive symptoms restricting social behaviour and thereby transmission of infectious agents. ${ }^{52}$ 
<smiles>N[C@@H](CC(=O)O)C(=O)Nc1ccccc1C(=O)C[C@@H](N)C(=O)O</smiles><smiles>Nc1c(O)cccc1C(=O)O</smiles>

3-Hydroxyanthranilic acid<smiles>NC(C(=O)O)=C(CCC=O)C(=O)O</smiles><smiles>CCCC</smiles><smiles>O=C(O)c1cccnc1C(=O)O</smiles>

Quinolinic acid

Figure 1: Indole-2,3-dioxygenase pathway.

3-HAO: 3-hydroxyanthranilic acid oxidase; IDO: indole-2,3-dioxygenase; KAT II: kynurenine aminotransferase II; KMO: kynurenine 3-monooxygenase.

Overexpressing this defence mechanism has the unfortunate consequence of also depleting not only available serotonin, but by extension melatonin, the monoamines most commonly associated with depressive symptoms and sleep disorders. This diversion may help explain the blunted response to selective serotonin reuptake inhibitors (SSRI) found in many patients with IBD, since SSRI drugs act downstream of neurotransmitter synthesis. While most studies of IDO1 are focussed on depletion of serotonin, it is important to note that melatonin is an important inhibitor of MMP-9,53 an upregulated enzyme that causes epithelial barrier damage. As such, depletion of melatonin likely plays a role in both intestinal and BBB permeability. Infliximab treatment strongly downregulates the expression of IDO1, and this may be a significant explanation for the antidepressant effects of anti-TNF treatment, as well as its effects on sleep quality. ${ }^{29}$

Additionally, in animal models, inhibition of IDO1 by 1-methyltryptophan had comparable 


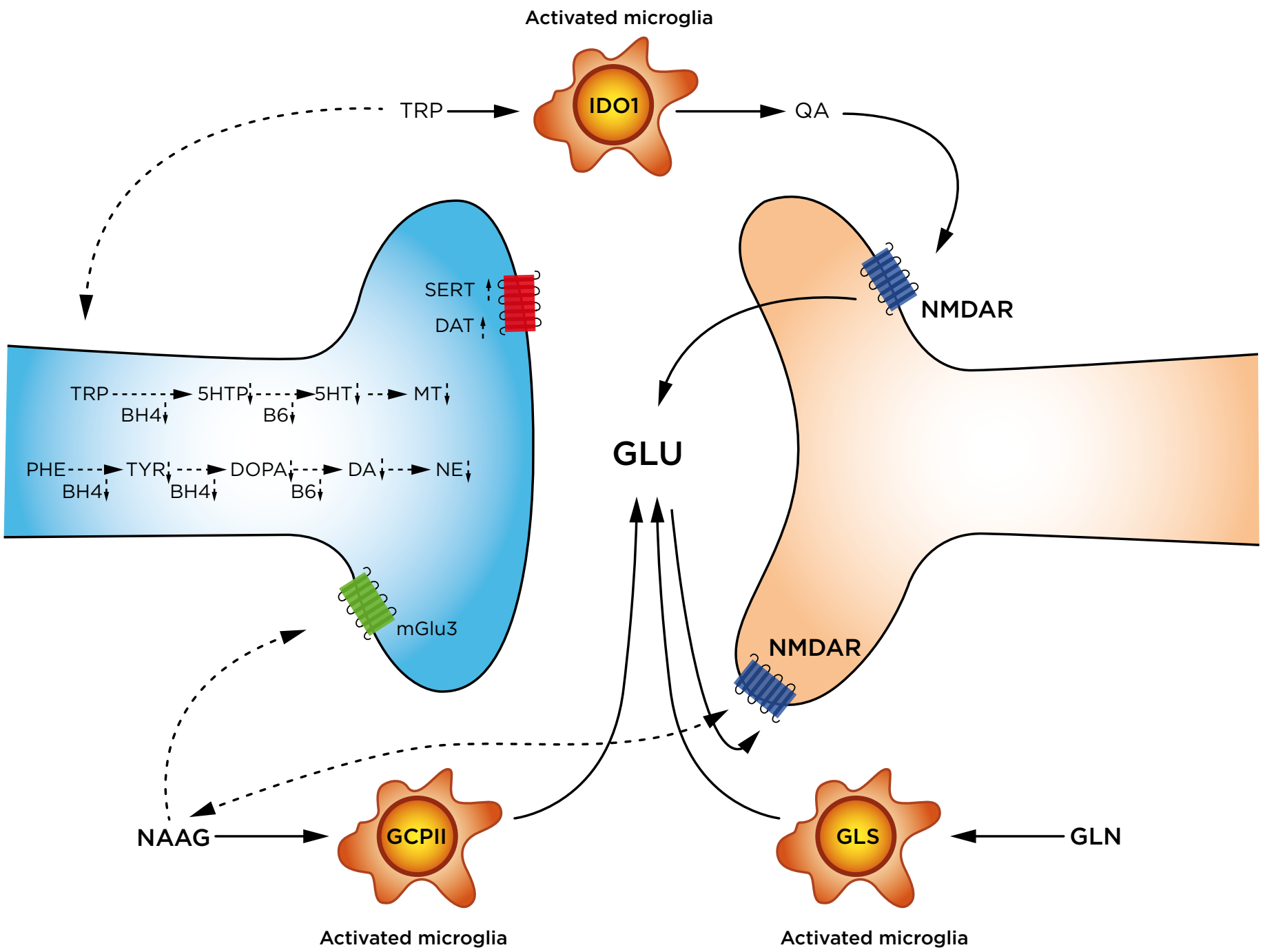

Figure 2: Inflammation-altered pathways in neurotransmission.

Synthesis of $5 \mathrm{HT}, \mathrm{MT}$, DA, and NE are slowed by lowered availability of $\mathrm{BH} 4$ and $\mathrm{B} 6$. $\mathrm{BH} 4$ is a cofactor in the hydroxylation of TRP, PHE, and TYR. B6 is a cofactor in the decarboxylation of DOPA to form DA, and the decarboxylation of 5HTP to form 5HT. Reuptake of DA by the DAT and of 5HT by the SERT are increased.

Activated microglia express three enzymes responsible for increased glutamate and glutamatergic signalling: 1) IDO1 catabolises TRP to QA. QA is an agonist at the NMDAR, leading to GLU release. 2) GCPII cleaves NAAG to N-acetylaspartate (not shown) and GLU. As a result, NAAG signalling at both the mGlu3 and the NMDAR are decreased. 3) GLS hydrolyses GLN to GLU. Removal of excess GLU by the GLT1/EAAT2 is also downregulated (not shown).

Impaired or downregulated pathways are shown with dotted arrows.

5HT: serotonin; 5HTP: 5-hydroxytryptophan; BH4: tetrahydrobiopterin; B6: pyridoxyl-5'-phosphate; DA: dopamine; DAT: dopamine transporter; DOPA: L-3,4-dioxyphenylalanine; EAAT2: excitatory amino acid transporter 2; GCPII: glutamate carboxypepsidase-II; GLN: glutamine; GLS: glutaminase; GLT1: glutamate transporter-I/; GLU: glutamate; IDO1: Indole-2,3-dioxygenase; mGlu3: metaboglutamate-3 receptor; MT: melatonin; NAAG: $\mathrm{N}$-acetylaspartylglutamate; NE: norepinephrine; NMDAR: N-methyl-D-aspartate receptor; PHE: phenylalanine; QA: quinolinic acid; SERT: serotonin transporter; TRP: tryptophan; TYR: tyrosine. 
effects on depressive behaviour to treatment with infliximab, suggesting that IDO1 may be a viable drug target for inflammation-mediated depression. ${ }^{54}$ There are multiple clinical trials investigating IDO1 inhibitors as add-on therapy for cancer treatments, so there may well be an approved drug in this class in the next several years.

\section{Glutamate-N-methyl-D-aspartate receptor}

As a continuation of the effects of IDO1, a primary catabolite of tryptophan is quinolinic acid (QA; Figure 1). QA is an N-methyl-Daspartate receptor (NMDAR) agonist, leading to increased glutamate signalling, release of additional glutamate, and inhibition of the release of dopamine. QA binds preferentially to NMDAR in the forebrain, in areas critical to mood, memory, and sleep regulation. ${ }^{55}$ Multiple studies have shown that the cognitive fatigue in IBD is postsynaptic in origin, and it therefore seems likely that NMDAR signalling plays a critical role. ${ }^{21}$ One of the purported sites for the rapid antidepressant action of ketamine in treatment-resistant depression is the NMDAR. In humans with treatment-resistant depression, low-dose ketamine as an add-on therapy dropped levels of both TNFa and IL-6, ${ }^{56}$ while one animal study showed it also lowered the levels of QA and microglia. ${ }^{57}$ It remains to be seen how effective ketamine will be in patients with IBD, but the studies above suggest a more complex and useful anti-inflammatory role in addition to its use as an antidepressant.

Excitotoxicity in the immediate term is most likely manifested by anhedonia and 'brain fog'. However, in the long term, excitotoxicity destroys neurons, specifically dopaminergic neurons. This destruction is exactly the type of process thought to lead to Parkinson's disease. Notably, IBD has long had a particularly strong association with development of Parkinson's disease, ${ }^{58}$ and NMDAR glutamate signalling could be a significant contributor. In support of this hypothesis, consider that IDO1 catabolites are increased in the cerebrospinal fluid of Parkinson's patients, ${ }^{59}$ and animal models of Parkinsonism are partially induced with $Q A .{ }^{60}$

Not only is glutamate release and signalling increased thanks to $Q A$, but also by upregulations of glutamate carboxypeptidase ${ }^{161}$ and glutaminase. ${ }^{62}$ By hydrolysing either $\mathrm{N}$-acetylaspartylglutamate or glutamine, additional glutamate is released, augmenting the glutamatergic (excitotoxic) responses. Increased glutamate is indeed visible in the brain by proton magnetic resonance spectroscopy. ${ }^{63}$ Along with increased release, signalling, and synthesis of glutamate, the primary reuptake transporter (EAAT2/GLT1) is downregulated. ${ }^{64}$ Added to that, decarboxylation of glutamate to GABA by GAD1 is likely regulated by $N F-\kappa B$, and requires vitamin $\mathrm{B} 6$ as a cofactor (see below). With these enzymes combined, glutamate excitotoxic signalling almost certainly plays a central role in the neuropsychiatric comorbidities of IBD.

\section{Tetrahydrobiopterin and Neopterin}

Along with upregulation of IDO1, cytokines also are associated with diversion of the catabolic synthesis of tetrahydrobiopterin (BH4), a critical cofactor for hydroxylation of phenylalanine, tyrosine, and tryptophan, the rate-limiting step en route to the catecholamines and serotonin/melatonin. Depletion of $\mathrm{BH} 4$ actually occurs in two ways. First, large upregulation of the first enzyme in the $\mathrm{BH} 4$ synthesis (GTP cyclohydrolase) creates a synthesis bottleneck, overwhelming the second enzyme in the pathway. ${ }^{65}$ As a consequence, the critical $\mathrm{BH} 4$ precursor is unhelpfully diverted to neopterin. Second, cytokines strongly induce nitric oxide synthase and reactive oxygen species, both of which require $\mathrm{BH} 4$ as a cofactor, ${ }^{66}$ depleting the available supply. Both processes decrease the availability of monoamine neurotransmitters, and are associated with anxiety, fatigue, and anhedonia. In one particularly elegant study by Felger et al.,67 reverse microdialysis of L-DOPA into the brains of rhesus monkeys reversed the symptoms of IFNa-induced depression, suggesting that hydroxylation (controlled by $\mathrm{BH} 4$ levels) was the dominant issue, and subsequent decarboxylation and vesicular transport (via VMAT2) might be functioning normally.

\section{Pyridoxyl-5'-Phosphate: Vitamin B6}

The final step in the synthesis of serotonin from 5-hydroxytryptophan, dopamine from L-DOPA, and GABA from glutamate is decarboxylation by the amino acid decarboxylases. As with the 
hydroxylases discussed above, these enzymes are dependent on the availability of a cofactor: in this case, pyridoxal-5'-phosphate (the active form of Vitamin B6), of which patients with IBD are frequently deficient.68 Studies on RA suggest that deficits in active $\mathrm{B} 6$ are not an absorption issue, but rather alterations in processing and metabolism. ${ }^{69}$ While there are considerably less data available, it has been reported that tryptophan metabolites inhibit the phosphorylation of pyridoxal. ${ }^{70} \mathrm{~A}$ recent animal study showed that NF-kB knockout mice had a downregulation of $A O X 1$, the principal metabolic enzyme responsible for converting pyridoxal-5'phosphate to pyridoxic acid. ${ }^{71}$ While this finding does not prove AOX1 is upregulated by $N F-\kappa B$, it certainly fits the pattern of NF- $\kappa B$ playing a central controlling role in inflammatory responses in the brain.

\section{CONCLUSIONS AND FINAL THOUGHTS}

Reflecting the mounting evidence, the American College of Gastroenterology (ACG), the British Society for Gastroenterology (BSG), the American Gastroenterological Association (AGA), the World Gastroenterology Organisation (WGO), and the Crohn's \& Colitis Foundation have all added some level of psychiatric assessment and management to their respective clinical guidelines. This is an important step toward successfully treating patients with IBD. However, as an example, the ACG recommendation (strong recommendation, very-low level of evidence) $)^{72,73}$ points out a larger problem in the need for broad screening. As an illustration, in a 2017 report, $>4,000$ patients were asked to self-evaluate, with only $20 \%$ of patients with Crohn's disease and $14 \%$ of patients with ulcerative colitis claiming to be depressed. However, by using a short questionnaire (Patient Health Questionnaire depression scale [PHQ-8]), an alarming 38\% of patients with Crohn's disease and 32\% of patients with ulcerative colitis in the same cohort met the criteria for depression. ${ }^{5}$ Given the antidepressant effects of some treatments (see above), the actual percentages in treatmentnaïve patients could be considerably higher. Since depression has such strong effects on motivation (including adherence with diagnostic and treatment protocols), ${ }^{74}$ devastating effects on disease progression, and significant increases to mortality, the need for psychiatric screening as part of disease management cannot be overstated.

There are two additional problems to be addressed in screening and reporting. First, the phrase "antidepressant usage" found in many studies is wholly insufficient. This phrase is comparable to using "IBD medication" in gastroenterology. Different antidepressant classes are as mechanistically dissimilar from each other as aminosalicylate and infliximab, possibly even more so. Given that some specific SSRI drugs (mirtazapine) are reported to worsen systemic inflammation, ${ }^{75}$ while at least one norepinephrine-dopamine reuptake inhibitor (bupropion) is reported to lessen it, ${ }^{76}$ such granular detail is critical.

Second, as mentioned in the introduction, there is a dearth of reliable biomarkers for depression and anxiety. As a result, diagnosis often relies on various questionnaires that are not entirely comparable. It would be wise of the advisory bodies of IBD treatment to recommend a diagnostic tool for depression alongside the aforementioned need for its diagnosis.

Using the statistics quoted above, roughly one in three patients with IBD is potentially depressed, and possibly as high as one in six has considered, or is considering, suicide as a result of that depression within the past year. ${ }^{77}$ For a typical gastroenterologist, that translates to seeing multiple patients per week that could be actively suicidal. Would their approach change if aware that the next patient was slowly (or rapidly) losing the battle against depression exacerbated by IBD? One very small scale report from the Jackson County, Missouri, USA, Medical Examiner's Office might provide some insight.78 Of 12 Crohn's patients that died from 2008 to 2010 (average age: 45 years), there were five suicides, three accidental drug overdoses, and one case of liver failure from alcohol abuse. Only two of 12 died of IBD complications. As the authors rightly point out, these numbers certainly will not scale to the whole population, and treatments have certainly improved in the past decade. However, this paper serves as a sobering reminder that the intestines are not the only battlefront for IBD patients, nor their caregivers. 


\section{References}

1. Crohn BB. Regional Ileitis (1949), New York: Grune \& Stratton.

2. Neuendorf R et al. Depression and anxiety in patients with inflammatory bowel disease: a systematic review. J Psychosom Res. 2016;87:70-80.

3. Nyuyki KD, Pittman QJ. Toward a better understanding of the central consequences of intestinal inflammation. Ann N Y Acad Sci. 2015;1351:149-54.

4. Canakis A, Qazi T. Sleep and fatigue in IBD: an unrecognized but important extra-intestinal manifestation. Curr Gastroenterol Rep. 2020;22(2):8

5. Kochar B et al. Depression is associated with more aggressive inflammatory bowel disease. Am J Gastroenterol. 2018;113(1):80-5.

6. Gradus JL et al. Inflammatory bowel disease and completed suicide in Danish adults. Inflamm Bowel Dis. 2010;16(12):2158-61.

7. Reichenberg A et al. Cytokineassociated emotional and cognitive disturbances in humans. Arch Gen Psychiatry. 2001;58(5):445-52.

8. Wright CE et al. Acute inflammation and negative mood: mediation by cytokine activation. Brain Behav Immun. 2005;19(4):345-50.

9. Vijaya Kumar $\mathrm{K}$ et al. Bacillus Calmette-Guérin vaccine induces a selective serotonin reuptake inhibitor (SSRI)-resistant depression like phenotype in mice. Brain Behav Immun. 2014;42:204-11.

10. Vasilyeva E et al. Serum cytokine profiles in children with Crohn's Disease. Mediators Inflamm. 2016;2016:7420127.

11. Miller $\mathrm{AH}$ et al. Cytokine targets in the brain: impact on neurotransmitters and neurocircuits. Depress Anxiety. 2013:30(4):297-306

12. Capuron $L$ et al. Neurobehavioral effects of interferon-alpha in cancer patients: phenomenology and paroxetine responsiveness of symptom dimensions. Neuropsychopharmacology. 2002;26(5):643-52

13. Asnis GM, De La Garza R 2nd. Interferon-induced depression in chronic hepatitis $\mathrm{C}$ : a review of its prevalence, risk factors, biology, and treatment approaches. J Clin Gastroenterol. 2006;40(4):322-35

14. Knowles SR et al. Preliminary examination of the relations between disease activity, illness perceptions, coping strategies, and psychological morbidity in Crohn's disease guided by the common sense model of illness. Inflamm Bowel Dis. 2011;17(12):2551-7.
15. Liu T et al. NF-кB signaling in inflammation. Signal Transduct Target Ther. 2017;2:17023.

16. Boston University. NF-kB target genes. Available at: https://www. bu.edu/nf-kb/gene-resources/targetgenes/. Last accessed: 17 July 2020.

17. Goldsmith DR et al. A meta-analysis of blood cytokine network alterations in psychiatric patients: comparisons between schizophrenia, bipolar disorder and depression. Mol Psychiatry. 2016;21(12):1696-709.

18. Enache D et al. Markers of central inflammation in major depressive disorder: a systematic review and meta-analysis of studies examining cerebrospinal fluid, positron emission tomography and post-mortem brain tissue. Brain Behav Immun. 2019;81:24-40.

19. Pandey GN et al. Proinflammatory cytokines in the prefrontal cortex of teenage suicide victims. J Psychiatr Res. 2012;46(1):57-63.

20. Janelidze $S$ et al. Cytokine levels in the blood may distinguish suicide attempters from depressed patients. Brain Behav Immun. 2011;25(2):335-9

21. Gray MA et al. Anti-TNFa therapy in IBD alters brain activity reflecting visceral sensory function and cognitive-affective biases. PLoS One. 2018;13(3):e0193542.

22. Schwartz JE et al. A Phase I trial of recombinant tumor necrosis factor (rTNF) administered by continuous intravenous infusion in patients with disseminated malignancy. Biotherapy. 1989;1(3):207-14.

23. Brown ER et al. A clinical study assessing the tolerability and biological effects of infliximab, a TNF-alpha inhibitor, in patients with advanced cancer. Ann Oncol. 2008;19(7):1340-6

24. Raison CL et al. A randomized controlled trial of the tumor necrosis factor antagonist infliximab for treatment-resistant depression: the role of baseline inflammatory biomarkers. JAMA Psychiatry. 2013;70(1):31-41.

25. Kappelmann $\mathrm{N}$ et al. Antidepressant activity of anti-cytokine treatment: a systematic review and metaanalysis of clinical trials of chronic inflammatory conditions. Mo Psychiatry. 2018;23(2):335-43.

26. Kaster MP et al. Depressive-like behavior induced by tumor necrosis factor-a in mice. Neuropharmacology. 2012;62(1):419-26.

27. Ting EY et al. Role of interleukin-6 in depressive disorder. Int $\mathrm{J} \mathrm{Mol} \mathrm{Sci.}$ 2020;21(6):2194

28. Rochfort KD et al. Tumour necrosis factor- $\alpha$-mediated disruption of cerebrovascular endothelial barrie integrity in vitro involves the production of proinflammatory interleukin-6. Neurochem. 2016:136(3):564-72.

29. Leal RF et al. Identification of inflammatory mediators in patients with Crohn's disease unresponsive to anti-TNFa therapy. Gut. 2015;64(2):233-42.

30. Menard $C$ et al. Social stress induces neurovascular pathology promoting depression. Nat Neurosci. 2017;20(12):1752-60.

31. Felger JC et al. What does plasma CRP tell us about peripheral and central inflammation in depression? Mol Psychiatry. 2020;25(6):1301-11.

32. Yohn SE et al. Effort-related motivational effects of the pro-inflammatory cytokine interleukin-6: pharmacological and neurochemical characterization. Psychopharmacology (Berl). 2016;233(19-20):3575-86.

33. Herman ML, Kane SV. Treatment nonadherence in inflammatory bowel disease: identification, scope, and management strategies. Inflamm Bowel Dis. 2015:21(12):2979-84.

34. Späth-Schwalbe E et al. Acute effects of recombinant human interleukin- 6 on endocrine and central nervous sleep functions in healthy men. J Clin Endocrinol Metab. 1998;83(5):1573-9.

35. Sun $Y$ et al. The effects of interleukin- 6 neutralizing antibodies on symptoms of depressed mood and anhedonia in patients with rheumatoid arthritis and multicentric Castleman's disease. Brain Behav Immun. 2017;66:156-64.

36. Gossec L et al. Fatigue in rheumatoid arthritis: quantitative findings on the efficacy of tocilizumab and on factors associated with fatigue. The French multicentre prospective PEPS Study. Clin Exp Rheumatol. 2015;33(5):664-70.

37. Milovanovic J et al. Interleukin-17 in chronic inflammatory neurological diseases. Front Immunol. 2020;11:947.

38. Liu Z et al. IL-17A exacerbates neuroinflammation and neurodegeneration by activating microglia in rodent models of Parkinson's disease. Brain Behav Immun. 2019;81:630-45.

39. Griffiths CEM et al. Impact of ixekizumab treatment on depressive symptoms and systemic inflammation in patients with moderate-tosevere psoriasis: an integrated analysis of three Phase 3 clinica studies. Psychother Psychosom. 2017:86(5):260-7.

40. Jha MK et al. Interleukin 17 selectively predicts better outcomes with bupropion-SSRI combination: novel $T$ cell biomarker for antidepressant medication selection. Brain Behav Immun. 2017:66:103-10. 
41. Zhang $X$ et al. [IL-17 and IL23 expression as a predictor of response to infliximab treatment in Crohn's disease]. Zhonghua Nei Ke Za Zhi. 2015;54(11):940-4. (In Chinese)

42. Borren NZ et al. Longitudinal trajectory of fatigue with initiation of biologic therapy in inflammatory bowel diseases: a prospective cohort study. J Crohns Colitis. 2020;14(3):309-15

43. Gordon KB et al. Anxiety and depression in patients with moderateto-severe psoriasis and comparison of change from baseline after treatment with guselkumab vs. adalimumab: results from the Phase 3 VOYAGE 2 study. J Eur Acad Dermatol Venereol. 2018;32(11):1940-9.

44. Stevens BW et al. Vedolizumab therapy is associated with an improvement in sleep quality and mood in inflammatory bowel diseases. Dig Dis Sci. 2017:62(1):197-206. [Erratum in Dig Dis Sci. 2017;62(2):552].

45. Bottomley JM et al. Vagus nerve stimulation (VNS) therapy in patients with treatment resistant depression: a systematic review and meta-analysis. Compr Psychiatry. 2019;98:152156. [epub ahead of print].

46. Yang $A C$ et al. Physiological bloodbrain transport is impaired with age by a shift in transcytosis. Nature. 2020:583(7816):425-30.

47. Pan W, Kastin AJ. The bloodbrain barrier: regulatory roles in wakefulness and sleep. Neuroscientist. 2017;23(2):124-36

48. Daneman R, Rescigno M. The gut immune barrier and the bloodbrain barrier: are they so different? Immunity. 2009;31(5):722-35

49. Calarge CA et al. Gut permeability and depressive symptom severity in unmedicated adolescents. J Affect Disord. 2019;246:586-94

50. da Fonseca AC et al. The impact of microglial activation on blood-brain barrier in brain diseases. Front Cell Neurosci. 2014;8:362

51. D'Mello C et al. Cerebral microglia recruit monocytes into the brain in response to tumor necrosis factor alpha signaling during peripheral organ inflammation. J Neurosci. 2009;29(7):2089-102.

52. Miller $\mathrm{AH}$, Raison $\mathrm{CL}$. The role of inflammation in depression: from evolutionary imperative to modern treatment target. Nat Rev Immunol. 2016;16(1):22-34

53. Alluri $\mathrm{H}$ et al. Melatonin preserves blood-brain barrier integrity and permeability via matrix metalloproteinase-9 inhibition. PLoS One. 2016;11(5):e0154427.
54. Liu YN et al. TNFa mediates stressinduced depression by upregulating indoleamine 2,3-dioxygenase in a mouse model of unpredictable chronic mild stress. Eur Cytokine Netw. 2015;26(1):15-25.

55. Amidfar $M$ et al. The role of NMDA receptor in neurobiology and treatment of major depressive disorder: evidence from translational research. Prog Neuropsychopharmacol Biol Psychiatry. 2019;94:109668.

56. Chen $\mathrm{MH}$ et al. Rapid inflammation modulation and antidepressant efficacy of a low-dose ketamine infusion in treatment-resistant depression: a randomized, doubleblind control study. Psychiatry Res. 2018;269:207-11.

57. Verdonk F et al. Microglial production of quinolinic acid as a target and a biomarker of the antidepressant effect of ketamine. Brain Behav Immun. 2019;81:361-73.

58. Park $\mathrm{S}$ et al. Patients with inflammatory bowel disease are at an increased risk of Parkinson's disease: a South Korean nationwide populationbased study. J Clin Med. 2019;8(8):1191.

59. Iwaoka K et al. Impaired metabolism of kynurenine and its metabolites in CSF of Parkinson's disease. Neurosci Lett. 2020;714:134576.

60. Ghorayeb I et al. Simultaneous intrastriatal 6-hydroxydopamine and quinolinic acid injection: a model of early-stage striatonigral degeneration. Exp Neurol. 2001:167(1):133-47.

61. Zhang T et al. An ileal Crohn's disease gene signature based on whole human genome expression profiles of disease unaffected ileal mucosal biopsies. PLoS One. 2012;7(5):e37139.

62. Takeuchi $\mathrm{H}$ et al. Tumor necrosis factor-alpha induces neurotoxicity via glutamate release from hemichannels of activated microglia in an autocrine manner. J Biol Chem. 2006:281(30):21362-8.

63. Lv K et al. Neurotransmitter alterations in the anterior cingulate cortex in Crohn's disease patients with abdominal pain: a preliminary MR spectroscopy study. Neuroimage Clin. 2018;20:793-9.

64. Clark IA, Vissel B. Excess cerebral TNF causing glutamate excitotoxicity rationalizes treatment of neurodegenerative diseases and neurogenic pain by anti-TNF agents. Neuroinflammation. 2016;13(1):236.

65. Huang A et al. Cytokine-stimulated GTP cyclohydrolase I expression in endothelial cells requires coordinated activation of nuclear factor-
kappaB and Stat1/Stat3. Circ Res. 2005;96(2):164-71

66. Neurauter $\mathrm{G}$ et al. Chronic immune stimulation correlates with reduced phenylalanine turnover. Curr Drug Metab. 2008:9(7):622-7.

67. Felger JC et al. Levodopa reverses cytokine-induced reductions in striatal dopamine release. Int J Neuropsychopharmacol. 2015;18(4):pyu084.

68. Weisshof R, Chermesh I. Micronutrient deficiencies in inflammatory bowel disease. Curr Opin Clin Nutr Metab Care. 2015;18(6):576-81.

69. Chiang EP et al. Abnormal vitamin $B(6)$ status is associated with severity of symptoms in patients with rheumatoid arthritis. Am J Med. 2003;114(4):283-7.

70. Ueland PM et al. Inflammation, vitamin $\mathrm{B} 6$ and related pathways. Mol Aspects Med. 2017;53:10-27.

71. Jurk D et al. Chronic inflammation induces telomere dysfunction and accelerates ageing in mice. Nat Commun. 2014;2:4172.

72. Lichtenstein GR et al. ACG clinical guideline: management of Crohn's disease in adults. Am J Gastroenterol. 2018;113(4):481-517. [Erratum in Am J Gastroenterol. 2018;113(7):1101].

73. Chapman BJ, Jones GB. Underscoring the biochemical overlap between Crohn's disease and depression. Am J Gastroenterol. 2019:114(5):827-8.

74. Chablani SV et al. depressive symptoms predict antitumor necrosis factor therapy noncompliance and healthcare utilization in patients with inflammatory bowel disease. Dig Dis Sci. 2018;63(9):2482-4.

75. Kast RE. Anti- and pro-inflammatory considerations in antidepressant use during medical illness: bupropion lowers and mirtazapine increases circulating tumor necrosis factoralpha levels. Gen Hosp Psychiatry. 2003;25(6):495-6.

76. Kane S et al. Crohn's disease remission on bupropion. Gastroenterology. 2003;125(4):1290.

77. Fuller-Thomson E, Sulman J. Depression and inflammatory bowel disease: findings from two nationally representative Canadian surveys. Inflamm Bowel Dis. 2006;12(8):697-707.

78. Carson HJ et al. Psychosocial complications of Crohn's disease and cause of death. J Forensic Sci. 2014:59(2):568-70. 